Supporting Information

\title{
Super-resolution imaging of self-assembled nanocarriers using quantitative spectroscopic analysis for cluster extraction
}

Janel L. Davis ${ }^{\text {a }}$, Yang Zhang a, Sijia Yi ${ }^{\text {a }}$, Fanfan Du ${ }^{\text {a }}$, Ki-Hee Song a , Evan A. Scott ${ }^{\text {a }}$, Cheng Sun ${ }^{\text {b }}$ and Hao F. Zhang ${ }^{\text {a, * }}$

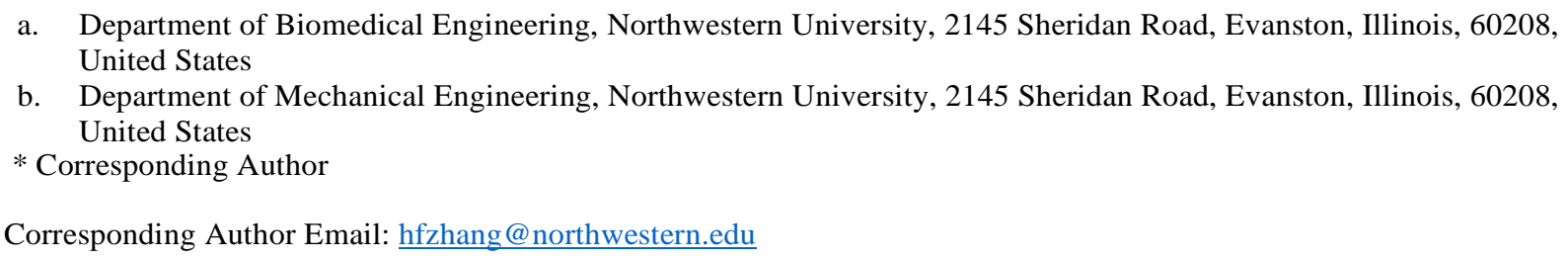

\begin{tabular}{|c|c|c|}
\hline \multicolumn{3}{|c|}{ Supporting Information Summary: 11 Pages, 15 Figures, \& 3 Notes } \\
\hline \multirow{2}{*}{$\begin{array}{l}\text { Page No. } \\
\quad \text { S2 }\end{array}$} & \multicolumn{2}{|r|}{ Supporting Information Table of Contents } \\
\hline & Figure S1 & $\begin{array}{l}\text { Bulk and single-molecule spectroscopic measurements of Nile Red in } \\
\text { polymersomes and immobilization reagents }\end{array}$ \\
\hline S3 & Figure S2 & $\begin{array}{l}\text { sPAINT reconstructions before and after applying the spectral window for } \\
\text { selecting polymersomes }\end{array}$ \\
\hline S3 & Figure S3 & $\begin{array}{l}\text { Comparison of the accuracy of quantifying polymersomes at different } \\
\text { localization densities }\end{array}$ \\
\hline S4 & Supporting Note 1 & Development of quantitative sPAINT \\
\hline S5 & Figure S4 & $\begin{array}{l}\text { Histograms of the spatial photon counts, spectral photon counts and spectral } \\
\text { precisions }\end{array}$ \\
\hline S5 & Figure S5 & Selection of the optimum parameters for density-based cluster analysis \\
\hline S6 & Figure S6 & Selection of the optimum parameters for quantitative sPAINT \\
\hline S6 & Figure S7 & Selection of the optimum parameters for counting polymersomes \\
\hline S7 & Figure S8 & $\begin{array}{l}\text { Super-resolution reconstruction of the polymersome sample using unutilized } \\
\text { localizations }\end{array}$ \\
\hline S7 & Figure S9 & The qSPACE workflow \\
\hline S8 & Supporting Note 2 & $\begin{array}{l}\text { Comparison of size measurements of polymersomes using sPAINT and } \\
\text { qSPACE }\end{array}$ \\
\hline S8 & Figure S10 & $\begin{array}{l}\text { The percent variance in size measurements and localization density for clusters } \\
\text { with increasing localizations per cluster }\end{array}$ \\
\hline S9 & Supporting Note 3 & Optimization of qSPACE for counting and sizing polymersomes \\
\hline S9 & Figure S11 & Threshold selection for detecting potential qSPACE clusters \\
\hline S10 & Figure S12 & Selection of the optimum spectral window for qSPACE \\
\hline S10 & Figure S13 & CryoTEM image of the polymersome sample \\
\hline S11 & Figure S14 & $\begin{array}{l}\text { Comparison of the size distributions of artifacts identified in control samples } \\
\text { for sPAINT and qSPACE }\end{array}$ \\
\hline S11 & Figure S15 & $\begin{array}{l}\text { Bulk spectroscopic measurements of Nile red in bovine albumin serum and } \\
\text { neutravidin }\end{array}$ \\
\hline
\end{tabular}





Figure S1: (a) Comparison of the average emission spectra of Nile Red in solutions containing polymersomes (PS) and sample preparation reagents (poly-L-lysine (PLL) and phosphate buffered saline (PBS)) measured using a fluorimeter and sPAINT. (b) Representative super-resolution reconstructions of the PLL coated glass used as the control sample. (Scale bar: $1 \mu \mathrm{m}$ ). (c-e) Histograms of the emission maxima ( $\left.\lambda_{\max }\right)$ of NR interactions in ROIs containing PS from three different images (six clusters were selected in each image). The selected spectral window (SW, $595-625 \mathrm{~nm}$ ) is highlighted in red. (f) Reproducibility test showing the histogram of $\lambda_{\max }$ from PS acquired on a different day with similar experimental conditions. (g) Comparison of the identification percent of localizations from the PS $\lambda_{\max }$ histogram and identification percent of localizations from the control $\lambda_{\max }$ histogram as the window size of the SW increases (peak position of SW = $610 \mathrm{~nm}$ ). 

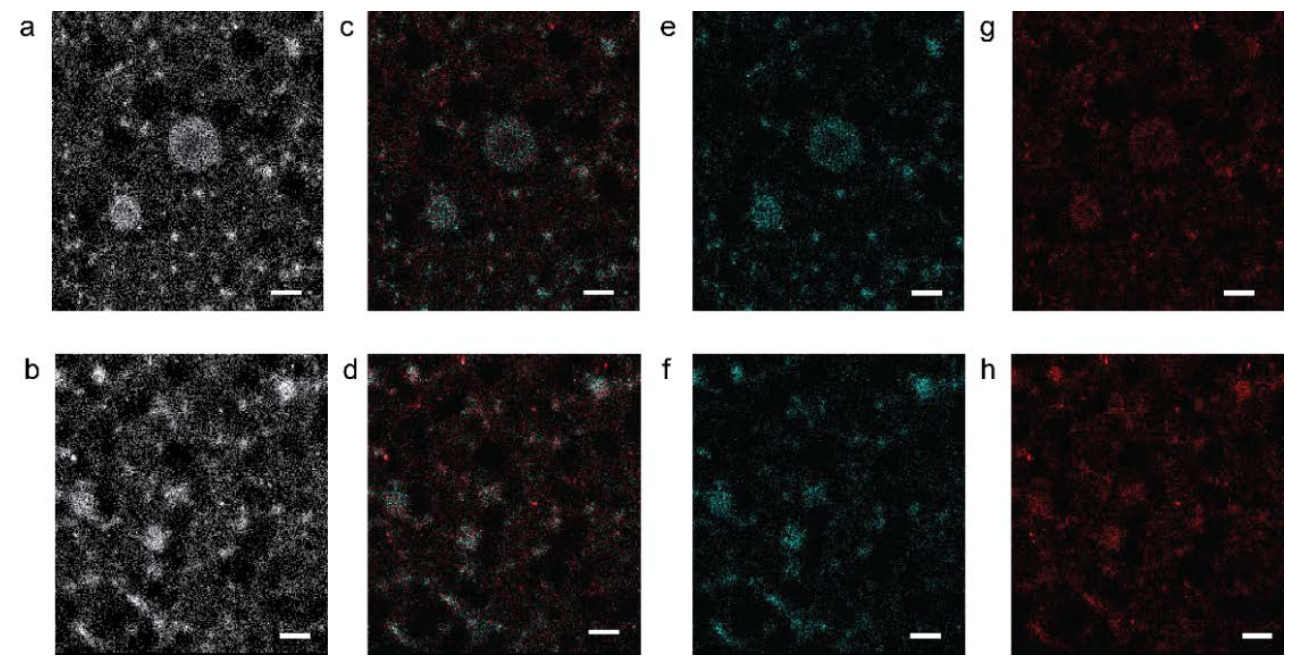

Figure S2: SPAINT reconstructions before applying the spectral window for selecting PS for (a) example 1 and (b) example 2. Overlay of the reconstructions with the localizations designated as PS in cyan and localizations designated from nonspecific binding in red from (c) example 1 and (d) example 2. sPAINT reconstructions of the PS channel for (e) example 1 and (f) example 2. sPAINT reconstructions of the nonspecific binding channels for (g) example 1 and (h) example 2 (Scale bar: $500 \mathrm{~nm}$ )


Figure S3: (a) Representative super-resolution reconstruction showing individual clusters with varying sizes identified by the optimized density-based spatial clustering for applications with noise (DBSCAN) algorithm marked by the red plus signs (Scale bar $500 \mathrm{~nm}$ ). (b) Comparison of the differences in the cluster counting accuracy using DBSCAN for trials using data with different cluster densities (number of nearest neighbors or number of $\mathrm{NN}$ ), the red bar shows data selected based on the spectroscopic and spatial information while the blue bar shows data selected for analysis based on spatial information alone. 


\section{Supporting Note 1}

We compared the performance of DBSCAN alone versus DBSCAN using localizations within the selected spectral window (SW). We used localizations with at least 450 photons in representative PS and control images for both trials. The photon distributions in the spatial and spectral domains are included in Figures S4a and S4b. Additionally, the distribution of the spectral precision (Figure S4c) shows that the spectral precision of a typical localization used in this study would be sufficient to be separated from the nonspecific binding with emission maxima populations $\sim 30 \mathrm{~nm}$ away from the emission maximum of the sample. We selected SW $(610 \pm 3 \mathrm{~nm})$ based on the histograms from the PS shown in Figures 2 and Figures S1c-e. We then selected a $30 \mathrm{~nm} \mathrm{SW}$ where the nonspecific binding to the PLL coated glass was minimized in the control sample (PLL coated glass with no PS) while maximizing the size of the window to allow as much NR interactions with PS to be included (Figure S1g). The selected SW rejected $83 \%$ of the localizations from the control sample while $60 \%$ of localizations associated with the PS were accepted. Changing the SW window size would affect the percentage localizations from both the nonspecific binding and the PS sample. For example, using a window size of $10 \mathrm{~nm}$, the percentage of localizations from nonspecific binding would be reduced to $5 \%$ and the percentage of localizations from the PS sample would be reduced to $23 \%$. This change would reduce the number of localizations per cluster in both cases causing inaccuracy in the size and morphology of the clusters using DBSCAN.

We used the selected cluster radius $(40 \mathrm{~nm})$ to determine the threshold on the minimum number of NN (min-NN) for each trial. For clarity, the min-NN threshold set based on DBSCAN alone is referenced by $\mathrm{NN}_{\mathrm{C}}$. Meanwhile, nearest neighbor thresholds based on SPAINT will be designated as $\mathrm{NN}_{\mathrm{V}}$. Figures S5a-c and S5d-f show the comparison between the histograms of NN for localizations in the control and PS samples with and without application of the SW. For the control and PS images the number of localizations was $\sim 10^{5}$ before the SW filter and $\sim 10^{4}$ the SW filter. By selecting localizations within the SW, the average number of NN reduced from 13 to 7 in the PS sample. Alternatively, in the control image the average number of NN reduced 5fold from 10 to 2 . This indicates that though the number of localizations was reduced for both groups, the application of the spectra-based criteria preferentially selected localizations which were members of clusters.

Based on this finding, a range of min-NN thresholds were tested for the defining clusters in the PS and control sample. For sPAINT a range of min-NNV from 5 to 20 were tested (Figure S5g). Meanwhile, for analysis based on DBSCAN alone, a range of min-NN $\mathrm{N}_{C}$ from 15 to 50 was tested (Figure S6a). A threshold was selected to achieve the minimal number of clusters in the control without causing morphology changes in the PS image (Figure S6b). Using this threshold, localizations with too few neighbors were rejected as noise. For sPAINT, a min- $\mathrm{NN}_{\mathrm{V}}$ of 10 was selected while a min- $\mathrm{NN}_{\mathrm{C}}$ of 20 was selected for DBSCAN alone. Figure S7 shows the application of the selected thresholds on an additional dataset with 42 and 31 identified before and after the SW filter respectively. 

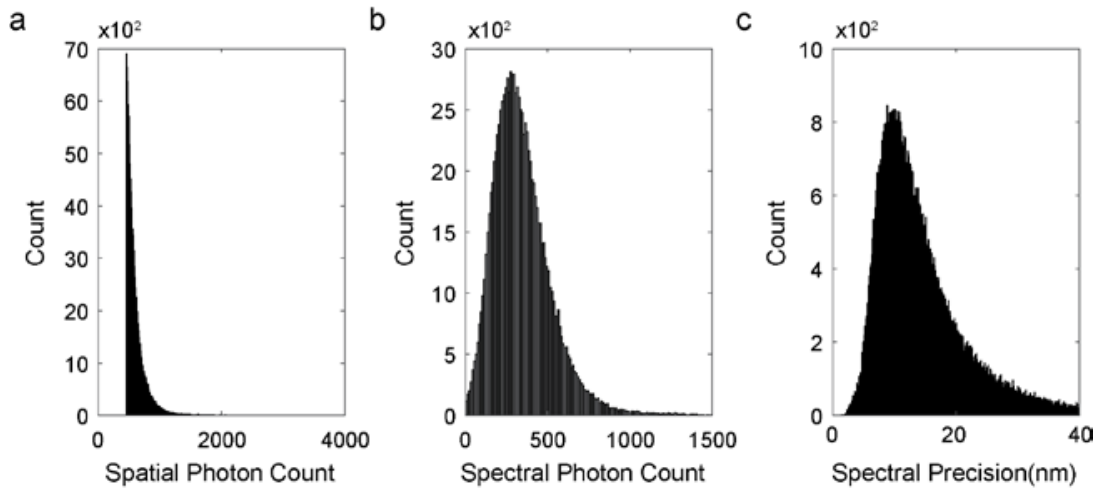

Figure S4: Histograms of the (a) spatial photon counts, (b) spectral photon counts and (c) spectral precision used for sSMLM analysis from a representative image.
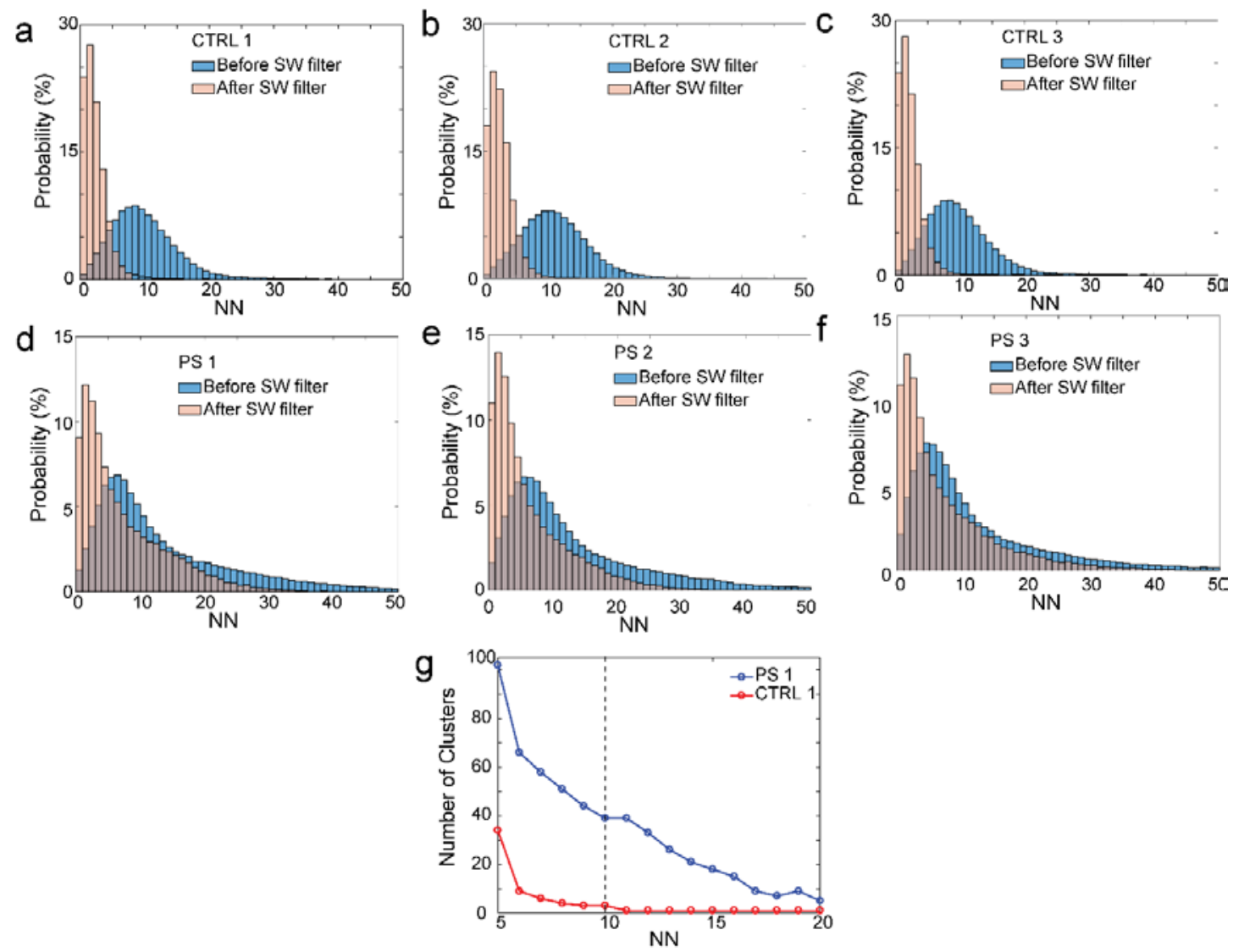

Figure S5: Histograms showing the comparison between the number of NN of localizations with 450 photons in the spatial domain before $\left(n=\sim 10^{5}\right)$ and after application of the selected spectral window ( $\mathrm{n}=\sim 10^{4}$ ) of (a-c) 3 control images and (d-f) 3 PS images. (g) Plots showing the number of clusters using localizations within the SW as the min-NN increases in representative PS and control images. The black dashed line shows the threshold used in this study. 

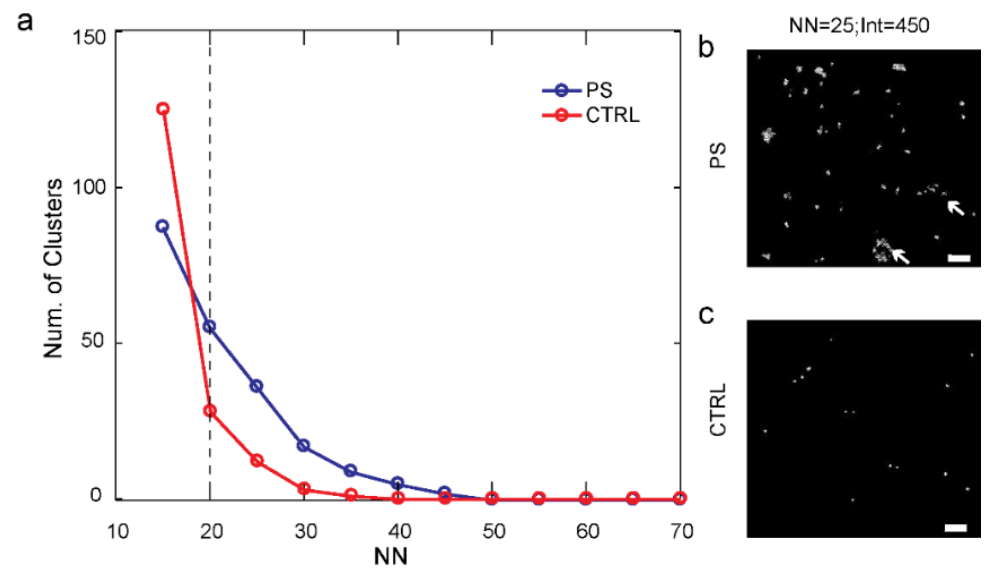

Figure S6: Plots showing the number of clusters with localizations with at least 450 photons in the spatial domain as the min-NN increases in the (a) PS and control images. The black dashed line shows the threshold used to compare spatial clustering without considering the PS window. Representative super-resolution reconstructions of the (b) PS sample (white arrows indicate changes in PS morphology) and (c) the control sample after application of the clustering thresholds $\left(\min -\mathrm{NN}_{\mathrm{C}}=25\right.$ and $\left.\mathrm{Int}=450\right)$. (Scale bar $\left.500 \mathrm{~nm}\right)$
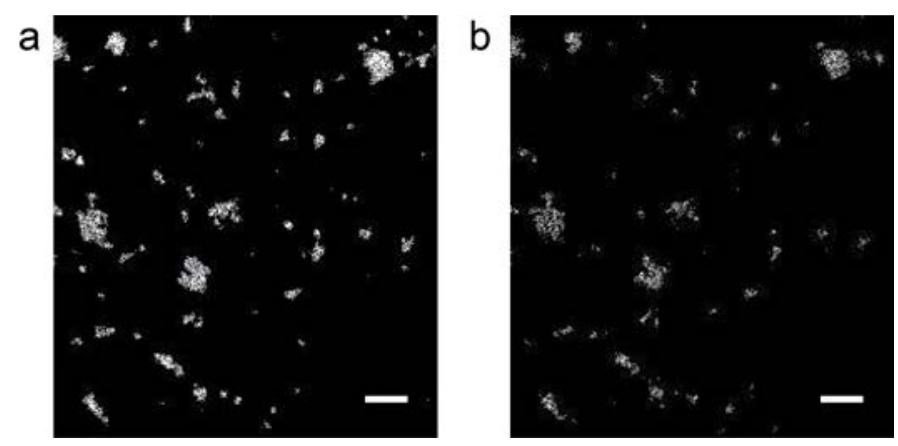

Figure S7: (a) Clusters extracted from a representative image of the PS sample based on DBSCAN alone; (b) Clusters extracted from the PS sample after the SW (Scale bar: $500 \mathrm{~nm}$ ) 


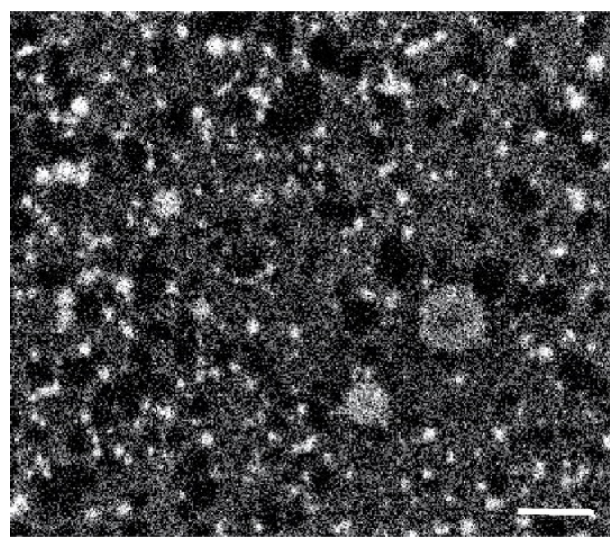

Figure S8: Localizations excluded from spectroscopic analysis based on photon number (Average localization uncertainty 25 nm, Photon budget: 300-450 in the spatial domain)

qSPACE Workflow

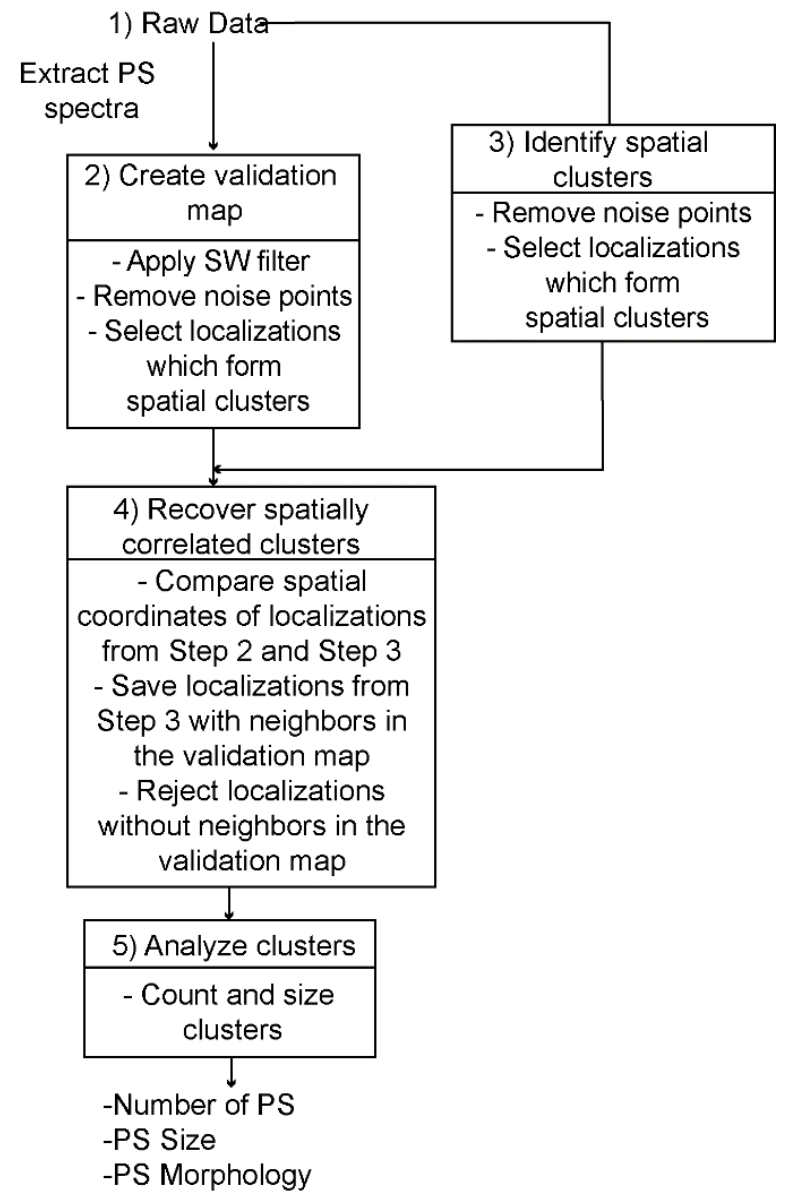

Figure S9: The detailed qSPACE workflow outlines how spectroscopic information is used to recover localizations from clusters from the PS sample while rejecting artifacts from nonspecific binding. 


\section{Supporting Note 2}

We measured the effect of the reduced number of localizations per cluster on size measurements by selecting 71 sPAINT clusters with at least 40 localizations. We calculated the percent variance $\left(\mathrm{P}_{\Delta \text { SIZE }}=\frac{\left|\overline{\mathrm{D}}-\mathrm{D}_{\mathrm{n}}\right|}{\overline{\mathrm{D}}}\right.$ where $\overline{\mathrm{D}}$ is the final diameter of the cluster and the $\mathrm{D}_{\mathrm{n}}$ is the estimated diameter for the cluster with $n$ localizations) for each cluster as the number of localizations sequentially increased. The average percent variance in cluster size reduced from $48 \%$ to $<5 \%$ as the number of localizations increased (blue line Figure S10). This trend was further investigated by grouping the analyzed clusters based on their varying sizes. We found that the majority of clusters smaller than $100 \mathrm{~nm}$ could be accurately sized with $\sim 25$ localizations per cluster. Meanwhile, clusters larger than $200 \mathrm{~nm}$ required 38 localizations per cluster for accurate sizing.

We accounted for the variation in the size requirements for clusters of different sizes by using a threshold based on the localization density ( $\mathrm{LD}=\frac{\mathrm{N}}{\pi \mathrm{r}^{2}}$ where $\mathrm{N}$ is the number of localizations in the cluster and $r$ is the radius of the cluster). The red line in Figure S10 represents the LD for an average PS (with $113 \mathrm{~nm}$ diameter) as the number of localizations increased. Using the results from the size variation measurements we set a $\mathrm{LD}$ threshold of $3.5 \times 10^{-3} \mathrm{~nm}^{-2}$ for accurate sizing.

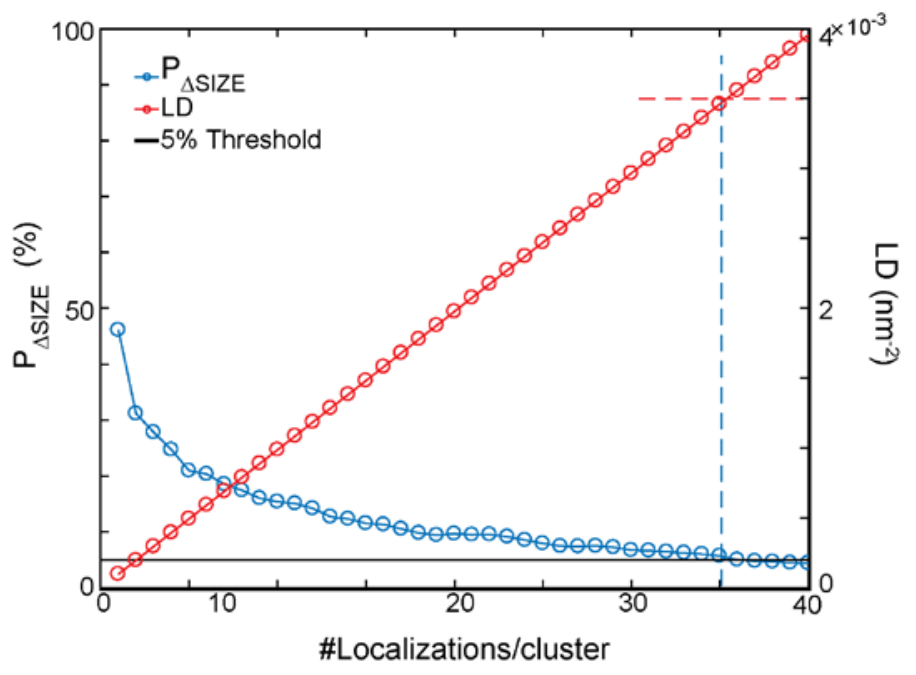

Figure S10: The average percent variance in size measurements $\left(\mathrm{P}_{\Delta \mathrm{SIZE}}\right)$ and localization density for clusters as the number of localizations per cluster increases. The black line shows where $\mathrm{P}_{\Delta \mathrm{SIzE}}$ $=5 \%$. The dashed blue and red lines indicate the min-NN of 35 and $\mathrm{LD}$ of $3.5 \times 10^{-3} \mathrm{~nm}^{-2}$ required for accurate sizing. 


\section{Supporting Note 3}

We tested the performance of qSPACE to reject artifacts from nonspecific binding and identify PS clusters. We used the clusters identified using the sPAINT criteria previously described (SW = [595-625], $\min -\mathrm{NN}_{\mathrm{v}}=10$ ) as a validation map. Potential PS clusters were identified using localizations using a min- $\mathrm{NN}_{\mathrm{c}}$ of 35 to limit variations in size measurements to $5 \%$ (Figures S11 \& S12). While traditional density-based algorithms would rely on stricter clustering threshold to remove potential artifacts, qSPACE uses a separate channel for sample validation. Using the validation channel, the specificity can be tuned by adjusting the min- $\mathrm{NN}_{\mathrm{v}}$ independently (Figure S12). The spatial coordinates of localizations in the validation map are compared to the spatial coordinates of localizations which formed potential clusters. Localizations which did not have neighbors in the validation map were rejected. All other localizations can be retained as members of true clusters. After validation, the min- $\mathrm{NN}_{\mathrm{C}}$ (35) for selecting the potential nanocarriers threshold set was used for cluster assignment.

a

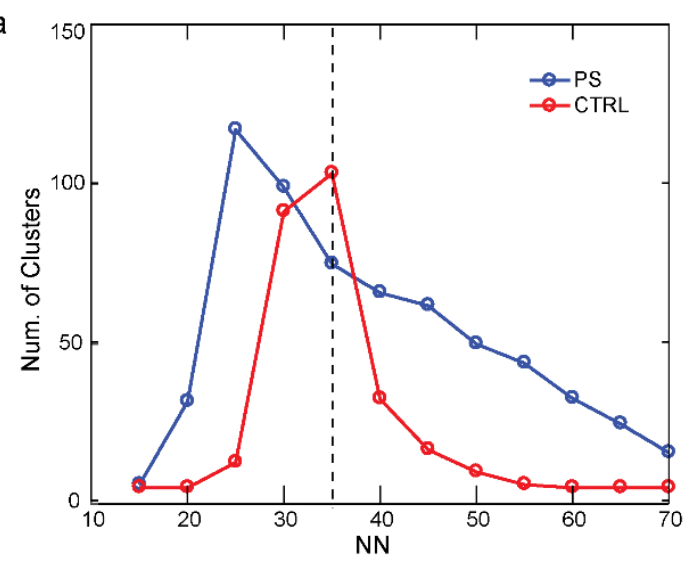

b

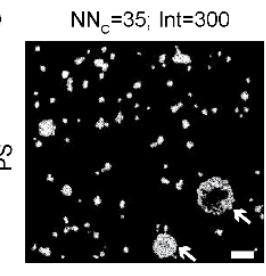

c

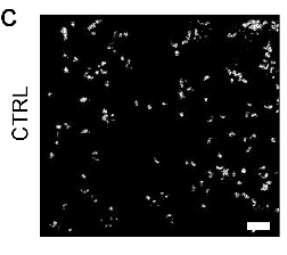

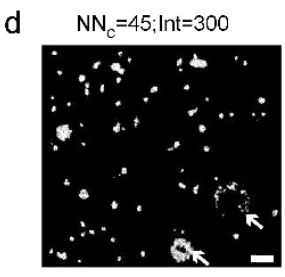

e



Figure S11: Plots showing the number of clusters at least 300 photons in the spatial domain as the minimum number of NN increases in the (a) PS and control images. The dashed black line represents the threshold used for detecting potential clusters for qSPACE. Representative superresolution reconstructions of the (b) PS sample and (c) the control sample after application of the clustering thresholds (min- $\mathrm{NN}_{\mathrm{C}}=35$ and Int=300), (d) PS sample and (e) the control sample after application of the clustering thresholds $\left(\min -\mathrm{NN}_{\mathrm{C}}=45\right.$ and $\left.\mathrm{Int}=300\right)$. The white arrows indicate morphology changes in d. (Scale bar: $500 \mathrm{~nm}$ ) 


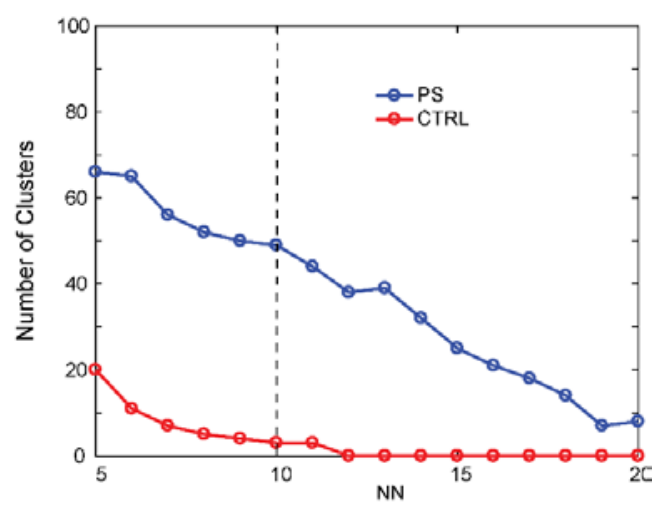

Figure S12: Plots showing the tuning of the min-NNv for noise removal in the validation channel using qSPACE with a SW window of $595-625 \mathrm{~nm}$ and $\min -\mathrm{NN}_{\mathrm{c}}$ of 35 for potential cluster assignment. The dashed black line shows the selected min- $\mathrm{NN}_{\mathrm{v}}$.

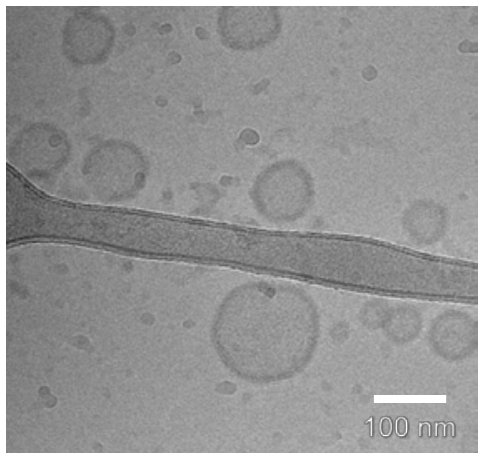

Figure S13: CryoTEM image of PS sample. 



Figure S14: (a) Histograms showing the size distribution of artifacts identified from 4 reconstructions of the control sample (809 clusters with average size $120 \pm 4 \mathrm{~nm}$ ) and clusters identified as artifacts in the LC (504 clusters with average size $111 \pm 5 \mathrm{~nm}$ ) and HC (501 clusters with average size $90 \pm 7 \mathrm{~nm}$ ) PS samples by qSPACE. (b) Histograms showing the size distribution of artifacts identified in 4 reconstructions of the control sample (229 clusters with average size $199 \pm 2 \mathrm{~nm}$ ) and clusters identified as artifacts in the LC (273 clusters with average size $220 \pm 11$ $\mathrm{nm}$ ) and HC (186 clusters with average size $211 \pm 8 \mathrm{~nm}$ ) PS samples by sPAINT (clusters formed from localizations with $\lambda_{\max }$ outside the SW filter) .



Figure S15: The average emission spectra of Nile Red in solutions containing PS and sample preparation reagents (bovine serum albumin (BSA) and Neutravidin (NEU)) measured using a fluorimeter. 